\title{
Influência da adiposidade sobre o risco inflamatório em pacientes com glicemia de jejum alterada
}

\author{
Influence of adiposity on inflammation risk in \\ patients with fasting glucose impairment
}

João Felipe MOTA ${ }^{1,2}$

Wilson Luvizotto MEDINA ${ }^{1,3}$

Fernando MORETO'

Roberto Carlos BURINI ${ }^{1}$

RE S U M O

\section{Objetivo}

Investigar as alterações dos marcadores inflamatórios em obesos com glicemia de jejum alterada.

\section{Métodos}

Foram avaliados 125 indivíduos adultos tendo sido pesquisados: Índice de Massa Corporal, circunferência da cintura, glicemia de jejum, proteína C reativa ultra-sensível, ácido úrico e homocisteína. Os grupos apresentando glicemia de jejum normal (grupo 1) e glicemia de jejum alterada (grupo 2) foram comparados entre si e em associação aos subgrupos de obesos, sobrepesos e eutróficos.

\section{Resultados}

O grupo 2 apresentou maiores valores de circunferência da cintura $(p<0,05)$, principalmente quando associados ao excesso de peso. Adicionalmente, o grupo 2 mostrou valores de proteína $C$ reativa e ácido úrico superiores, sendo os dos obesos maiores que os com sobrepeso e os eutróficos, enquanto a homocisteína foi semelhante entre obesos, com sobrepeso e eutróficos. O Índice de Massa Corporal correlacionou-se positivamente com ambos ácido úrico $(r=0,39, p<0,01)$ e proteína $C$ reativa $(r=0,37 ; p<0,01)$. A circunferência da cintura apresentou correlação apenas com o ácido úrico $(r=0,53, p<0,01)$. Porém, a correlação entre Índice de Massa Corporal e proteína $C$ reativa foi significante no grupo $2(r=0,66, p<0,01)$, mas não no grupo $1(r=0,25, p>0,05)$. 0 mesmo resultado foi encontrado em relação à circunferência da cintura e à proteína $C$ reativa, que se correlacionaram significantemente somente na presença de glicemia de jejum alterada $(r=0,40, p<0,05)$. Na regressão logística, a circunferência da cintura foi a variável explicativa $(11 \%, p<0,01)$ da alteração da glicemia de jejum.

\footnotetext{
1 Universidade Estadual Paulista, Faculdade de Medicina de Botucatu, Departamento de Saúde Pública, Centro de Metabolismo em Exercício e Nutrição. Distrito de Rubião Júnior, s/n., 18618-970, Botucatu, SP, Brasil. Correspondência para/Correspondence to: J.F. MOTA. E-mail: <joao.mota@inecs.com.br>.

2 Universidade São Francisco, Curso de Nutrição, Departamento de Nutrição. Bragança Paulista, SP, Brasil.

${ }^{3}$ Universidade Federal de São Carlos, Curso de Medicina, Departamento de Medicina. São Carlos, SP, Brasil.
} 
352 | J.F. MOTA et al.

\section{Conclusão}

Os estados pró-oxidativo e pró-inflamatório estiveram significantemente associados à glicemia de jejum alterada na presença de adiposidade corporal.

Termos de indexação: Diabetes mellitus. Estresse oxidativo. Inflamação. Obesidade.

\section{A B S T R A C T}

\section{Objective}

The objective of this study was to investigate changes in inflammatory markers in obese individuals with impaired fasting glucose.

\section{Methods}

The following data of 125 adult individuals were assessed: body mass index, waist circumference, fasting glucose, high-sensitivity c-reactive protein, uric acid and homocysteine. The groups with normal fasting glucose (group 1) and impaired fasting glucose (group 2) were compared with each other and with their subgroups, which consisted of obese, overweight and normal-weight individuals.

\section{Results}

Group 2 presented greater waist circumferences $(p<0.05)$, especially when associated with excess weight. Additionally, group 2 had higher C-reactive protein and uric acid values than group 1 and the obese in group 2 had higher C-reactive protein and uric acid than the overweight and normal-weight individuals of the same group. On the other hand, homocysteine levels were similar in all individuals, regardless of weight. Body mass index correlated positively with both uric acid $(r=0.39, p<0.01)$ and $C$-reactive protein $(r=0.37, p<0.01)$. Waist circumference correlated only with uric acid $(r=0.53, p<0.01)$. However, the correlation between body mass index and C-reactive protein was significant in group $2(r=0.66, p<0.01)$ but not in group $1(r=0.25, p>0.05)$. The same occurred regarding waist circumference and C-reactive protein, which correlated significantly only in the presence of impaired fasting glucose $(r=0.40, p<0.05)$. In logistic regression, waist circumference was the independent variable $(11 \%, p<0.01)$ of impaired fasting glucose.

\section{Conclusion}

Pro-oxidative and pro-inflammatory states were significantly associated with impaired fasting glucose in the presence of body fat.

Indexing terms: Diabetes mellitus. Oxidative stress. Inflammation. Obesity.

\section{N T R O D U ÇÃ O}

A resistência à insulina (RI) tem sido cada vez mais reconhecida como estado de inflamação crônica, apresentando similaridades com o processo de aterosclerose ${ }^{1}$. O efeito patogênico dessas doenças, provavelmente associado aos ácidos graxos livres, é mediado pelo aumento do estresse oxidativo e pela subseqüente inflamação ${ }^{2,3}$. A homocisteína (Hcy), um dos marcadores desse estresse, aumenta o risco de morte em portadores de diabetes mellitus tipo 2 (DM2), fato que pode ser atribuído ao prejuízo na função endotelial e na microcirculação coronariana ${ }^{4}$.

Uma relação direta entre Hcy e ácido úrico (AcUr) é observada em portadores de ateroscle- rose, refletindo o estresse oxidativo. Adicionalmente, o aumento das concentrações de AcUr parece estar relacionado à hiperinsulinemia. A hiperinsulinemia ativa o sistema renina-angiotensina, aumentando a angiotensina II, que eleva o AcUr plasmático tanto pela reabsorção de urato como pelo aumento de sua síntese a partir do metabolismo das purinas. Além disso, o hiperinsulinismo promove maior produção de espécies reativas ao oxigênio e ao nitrogênio por indução endógena de $\mathrm{NAD}(\mathrm{P}) \mathrm{H}$ (nicotinamida adenina dinucleotídeo-P) oxidase e xantina oxidase ${ }^{5}$.

O ácido úrico, em elevadas concentrações, induz o fator nuclear Kappa B e a proteína quimioatrativa de monócitos, mecanismos envolvidos 
no processo inflamatório presente na resistência à insulina e na aterosclerose ${ }^{6}$. Olexa et al. ${ }^{7}$ mostraram associação positiva entre o ácido úrico e o fator de necrose tumoral alfa, concluindo que o AcUr pode refletir ativação do estresse inflamatório por induzir células mononucleares a produzir citocinas, dentre elas a interleucina-6, precursora da proteína $\mathrm{C}$ reativa ${ }^{5}$.

O estresse inflamatório tem sido amplamente estudado em portadores de resistência à insulina, principalmente no que diz respeito aos riscos cardiovasculares, como a formação da placa ateromatosa. Em diversos estudos prospectivos foi verificada associação positiva entre o desenvolvimento da Rl e da síndrome metabólica e os marcadores inflamatórios como a proteína $C$ reativa ultra-sensível (PCR-us), sugerindo o papel do processo inflamatório na gênese do $\mathrm{DM} 2^{8,9}$.

A elevação das concentrações de PCR-us também está associada à obesidade, tanto em crianças quanto em adultos ${ }^{10}$. O tecido adiposo visceral seria o principal órgão secretor de citocinas pró-inflamatórias, as quais estimulam a liberação, pelo hepatócito, de proteínas da fase aguda, dentre elas a PCR e os fatores pró-trombóticos como o inibidor da ativação do plasminogênio (PAl-1) $)^{11}$.

A natureza da associação da PCR-us à RI ainda é pouco entendida ${ }^{12}$. Uma das hipóteses é de que a insensibilidade à insulina e/ou a hiperinsulinemia são conseqüências do excesso de gordura corporal que aumenta a descarga adrenérgica, promovendo maior lipólise e utilização dos ácidos graxos como principal substrato energético, reduzindo a captação de glicose ${ }^{13}$.

Desse modo, a perda de peso induziria, significativamente, a diminuição nas concentrações de PCR-us e AcUr, melhorando a síndrome da resistência insulínica ${ }^{5}$.

Assim, o presente estudo foi conduzido para investigar as associações entre estado glicêmico e as variações de indicadores pró-inflamatório e oxidante sob a influência da obesidade.

\section{M É TOD O S}

Em estudo transversal foram avaliados 125 indivíduos voluntários de ambos os sexos (72,8\% sexo feminino) com média de idade de 56 anos (desvio-padrão - DP=9). Os indivíduos foram divididos de acordo com a glicemia de jejum: normal $(\mathrm{G} 1:<100 \mathrm{mg} / \mathrm{dL})$ e alterada $(\mathrm{G} 2: \geq 100 \mathrm{mg} / \mathrm{dL})^{14} \mathrm{e}$ o Índice de Massa Corporal (IMC) ${ }^{15}$.

O estudo foi conduzido em 2006, na cidade de Botucatu (SP), dentro dos padrões exigidos pela Declaração de Helsinki e aprovado pela comissão de ética em pesquisa da Faculdade de Medicina de Botucatu, Brasil (protocolo $n^{\circ}$ 1812/2005). Todos os indivíduos assinaram o termo de consentimento livre e esclarecido informando-os sobre a proposta e os procedimentos do programa.

A avaliação da composição corporal constou da verificação da circunferência da cintura (CC), medida com fita métrica inextensível, do peso e da estatura, aferidos por balança antropométrica digital, para cálculo do $\mathrm{IMC}^{15}$.

Para análise das variáveis bioquímicas foi colhido sangue venoso após jejum de 12 horas. As concentrações plasmáticas de AcUr, glicose, lipídios e frações foram determinadas pelo método enzimático colorimétrico em analisador automático System Vitros Chemistry 950 Xrl (Johnson \& Johnson $\left.^{\circledR}\right)$. Para determinação das concentrações de lipoproteína de alta densidade - LDL-c foi utilizada a fórmula proposta por Friedewald et al. ${ }^{16}$. A PCR-us foi analisada por quimioluminescência (Immulite $\left.{ }^{\circledR}\right)$ com kits da DPC MedLab ${ }^{\circledR}$. As concentrações de Hcy foram determinadas por cromatografia líquida de alto desempenho (HPLC) ${ }^{17}$.

Os resultados das variáreis paramétricas foram expressos sob a forma de média e DP, acrescidos de mediana semi-amplitude interquartílica, quando a variável não tinha distribuição normal. Para comparação das variáveis paramétricas entre dois grupos, foram realizados o teste $t$ de Student para amostras independentes, ou o teste de Mann-Whitney. Para a análise de três ou mais grupos foi utilizada a análise de variância de duas vias ANOVA/MANOVA (pos hoc Duncan's test) ou 
Kruskal-Wallis ANOVA. Foram realizadas correlações de Pearson, com o intervalo de confiança de $95 \%$, para verificar as associações entre os indicadores antropométricos e os estados pró-oxidante e inflamatório. Também foi realizada uma regressão logística multivariada em stepwise, considerando a glicemia de jejum alterada como variável dependente. O nível de significância adotado foi $5 \%$. Todas as análises foram realizadas utilizando o software Statistica for Windows (version 5.1, Statsoft, Tulsa, USA).

\section{RESULTADOS}

Após a divisão dos grupos de acordo com a glicemia de jejum, os indivíduos com glicemia de jejum alterada (G2) apresentaram maior circun- ferência de cintura e concentrações superiores de triglicerídios, PCR-us e de AcUr, associadamente às menores concentrações de lipoproteína de alta densidade - HDL-colesterol (Tabela 1).

Quando os grupos foram subdivididos de acordo com a classificação do IMC, os subgrupos G1 e G2 não diferiram entre si (Tabela 2). Porém, ao analisar a CC os obesos do G2 apresentaram maior adiposidade abdominal, quando comparados aos obesos do $\mathrm{G} 1$ (111 DP=7,5 versus 102 $D P=13 \mathrm{~cm}, p<0,05)$, como mostra a Tabela 2.

As concentrações de PCR-us foram estatisticamente superiores $(p<0,05)$ apenas nos obesos do G2, quando comparadas a todos os outros subgrupos do G1 e G2 (Tabela 2). O AcUr acompanhou a PCR-us, os obesos do G2 apresentaram maiores concentrações quando compa-

Tabela 1. Comparação entre os grupos de indivíduos adultos com glicemia de jejum alterada e normal. Botucatu (SP), 2006.

\begin{tabular}{|c|c|c|c|c|c|}
\hline \multirow{2}{*}{ Variáveis } & \multicolumn{2}{|c|}{ Glicemia de jejum normal $(n=93)$} & \multicolumn{2}{|c|}{ Glicemia de jejum alterada $(n=32)$} & \multirow{2}{*}{$p^{*}$} \\
\hline & M & DP & M & DP & \\
\hline IMC $\left(\mathrm{kg} / \mathrm{m}^{2}\right)$ & 28,60 & 4,90 & 29,50 & 4,80 & 0,215 \\
\hline $\mathrm{CC}(\mathrm{cm})$ & 91,30 & 13,30 & 99,10 & 12,40 & $<0,010$ \\
\hline Colesterol total $(\mathrm{mg} / \mathrm{dL})$ & 216,30 & 38,90 & 209,00 & 39,50 & 0,391 \\
\hline $\mathrm{HDL}-\mathrm{c}(\mathrm{mg} / \mathrm{dL})$ & 53,90 & 13,90 & 47,90 & 12,30 & $<0,050$ \\
\hline LDL-c (mg/dL) & 133,20 & 37,50 & 123,00 & 34,10 & 0,193 \\
\hline Triacilglicerol (mg/dL) & 145,90 & 63,20 & 189,70 & 78,40 & $<0,010$ \\
\hline Homocisteína $(\mu \mathrm{M} / \mathrm{L})$ & 13,90 & 4,80 & 14,30 & 4,90 & 0,692 \\
\hline PCR- us $(\mathrm{mg} / \mathrm{dL})^{+}$ & 0,26 & 0,18 & 0,39 & 0,31 & $<0,050$ \\
\hline Ácido úrico $(\mathrm{mg} / \mathrm{dL})$ & 5,10 & 4,80 & 6,00 & 1,60 & $<0,004$ \\
\hline
\end{tabular}

CR-us: proteína C reativa ultra-sensível; CC: circunferência da cintura. Dados expressos em média (M), desvio-padrão (DP); IMC: índice de massa corporal. Mediana semi-amplitude interquartílica.

* Diferenças entre grupos: teste $t$ Student (amostras paramétricas) e Mann-Whitney U Test (amostras não-paramétricas), sendo as significantes expressas em negrito.

Tabela 2. Comparação de indicadores antropométricos e estresses oxidativo e inflamatório entre os grupos de adultos eutróficos, com sobrepeso e obesos, na presença ou na ausência de glicemia de jejum alterada. Botucatu (SP), 2006.

\begin{tabular}{|c|c|c|c|c|c|c|c|c|c|c|c|c|}
\hline \multirow{3}{*}{ Variáveis } & \multicolumn{4}{|c|}{$\begin{array}{c}\text { Eutrofia } \\
\left(\text { IMC: } 18,5-24,9 \mathrm{~kg} / \mathrm{m}^{2}\right)\end{array}$} & \multicolumn{4}{|c|}{$\begin{array}{c}\text { Sobrepeso } \\
\left(\mathrm{IMC}: 25-29,9 \mathrm{~kg} / \mathrm{m}^{2}\right)\end{array}$} & \multicolumn{4}{|c|}{$\begin{array}{c}\text { Obesidade } \\
\left(\mathrm{IMC}>30 \mathrm{~kg} / \mathrm{m}^{2}\right)\end{array}$} \\
\hline & \multicolumn{2}{|c|}{ G1 $(n=25)$} & \multicolumn{2}{|c|}{$\mathrm{G} 2(\mathrm{n}=7)$} & \multicolumn{2}{|c|}{$\mathrm{G} 1(\mathrm{n}=38)$} & \multicolumn{2}{|c|}{$\mathrm{G} 2(\mathrm{n}=13)$} & \multicolumn{2}{|c|}{$\mathrm{G} 1(\mathrm{n}=30)$} & \multicolumn{2}{|c|}{$\mathrm{G} 2(\mathrm{n}=12)$} \\
\hline & $M$ & $\mathrm{DP}$ & $\mathrm{M}$ & $\mathrm{DP}$ & M & DP & M & $\mathrm{DP}$ & $\mathrm{M}$ & $\mathrm{DP}$ & $M$ & $\mathrm{DP}$ \\
\hline$C C(\mathrm{~cm})$ & 78,40 & $8,10^{a}$ & 81,60 & $5,50^{\mathrm{a}}$ & 91,10 & $7,50^{b}$ & 94,5 & $5,4^{b}$ & 102,0 & 13,0 & 111,00 & $7,5^{d}$ \\
\hline Hcy $(\mu \mathrm{M} / \mathrm{L})$ & 12,40 & 2,30 & 11,90 & 3,30 & 14,20 & 5,80 & 12,8 & 2,7 & 14,7 & 4,7 & 15,20 & 4,2 \\
\hline PCR-us $(\mathrm{mg} / \mathrm{dL})^{+}$ & 0,13 & $0,11^{a}$ & 0,38 & $0,13^{a}$ & 0,26 & $0,16^{a}$ & 0,3 & $0,09^{a}$ & 0,4 & $0,20^{a}$ & 0,76 & $0,28^{b}$ \\
\hline AcUr (mg/dL) & 4,90 & $1,20^{a}$ & 4,70 & $1,90^{\mathrm{a}}$ & 4,90 & $1,20^{a}$ & 5,9 & $1,3^{a b}$ & 5,6 & $1,6^{\mathrm{ab}}$ & 6,30 & $1,5^{b}$ \\
\hline
\end{tabular}

G1: glicemia de jejum normal; G2: glicemia de jejum alterada; IMC: índice de massa corporal; CC: circunferência da cintura; Hcy: homocisteína; PCR-us: proteína C reativa ultra-sensível; AcUr: ácido úrico; M: Média; DP: desvio-padrão.

+ Mediana semi-amplitude interquartílica. Letras diferentes indicam diferenças inter e intragrupos $(p<0,05)$ - ANOVA/MANOVA (2-way, pos hoc Duncan's test) ou Kruskal-Wallis ANOVA. 
Tabela 3. Correlação entre os indicadores antropométricos e o estresse oxidativo e inflamatório estudados na presença ou ausência de glicemia de jejum alterada de adultos. Botucatu (SP), 2006.

\begin{tabular}{|c|c|c|c|c|c|c|}
\hline \multirow{2}{*}{ Variáveis } & \multicolumn{3}{|c|}{ IMC $\left(\mathrm{kg} / \mathrm{m}^{2}\right)$} & \multicolumn{3}{|c|}{$C C(\mathrm{~cm})$} \\
\hline & $\mathrm{G} 1+\mathrm{G} 2$ & G1 & $\mathrm{G} 2$ & $\mathrm{G} 1+\mathrm{G} 2$ & G1 & G2 \\
\hline PCR-us (mg/dL) & $0,37 *$ & 0,25 & $0,66^{*}$ & 0,22 & 0,09 & $0,40 *$ \\
\hline AcUr (mg/dL) & $0,39 *$ & $0,37 *$ & $0,37 *$ & $0,53^{*}$ & $0,53^{*}$ & 0,41 * \\
\hline
\end{tabular}

IMC: índice de massa corporal; CC: circunferência da cintura; G1: glicemia de jejum normal; G2: glicemia de jejum alterada; PCR-us: proteína C reativa ultra-sensível; AcUr: ácido úrico. ${ }^{*} p<0,05$.

Tabela 4. Regressão logística multivariada para determinar as variáveis associadas à glicemia de jejum alterada de indivíduos adultos. Botucatu (SP), 2006.

\begin{tabular}{lccc}
\hline Variáveis & Coeficiente $\beta$ & Erro padrão $\beta$ & $p^{*}$ \\
\hline IMC & $-0,152$ & 0,110 & 0,169 \\
CC & 0,324 & 0,112 & $\mathbf{0 , 0 0 4}$ \\
PCR-us & 0,083 & 0,063 & 0,187 \\
AcUr & $-0,087$ & 0,063 & 0,460 \\
Hcy & $-0,021$ & 0,097 & 0,828 \\
\hline
\end{tabular}

$R^{2}$ ajustado= 0,111.

IMC: índice de massa corporal; CC: circunferência da cintura; PCR-us: proteína C reativa ultra-sensível; AcUr: ácido úrico; Hcy: homocisteína.

*Valores significantes em negrito.

rados aos do $G 1$ e aos eutróficos do $G 2$, respectivamente $(6,3 \mathrm{DP}=1,5$ vs $4,9 \mathrm{DP}=1,2 \mathrm{mg} / \mathrm{dL} ; 6,3$ $D P=1,5$ vs $4,7 D P=1,9 \mathrm{mg} / \mathrm{dL}$ ) e aos sobrepesos do $G 1$ (6,3 DP=1,5 vs 4,9 DP=1,2mg/dL), segundo a Tabela 2. As concentrações de Hcy não diferiram tanto inter quanto intragrupos (Tabela 2).

Independentemente da glicemia de jejum alterada, houve correlação positiva significante entre o IMC, PCR-us $(r=0,37, p<0,01)$ e o ácido úrico $(r=0,39, p<0,01)$. Em relação à circunferência da cintura, esta apresentou correlação apenas com o ácido úrico $(r=0,53, p<0,001)$, como mostra a Tabela 3.

Quando analisadas estas correlações na ausência da glicemia de jejum alterada apenas aquelas relacionadas ao ácido úrico foram significantes (IMC x AcUr: $r=0,37 ; C C \times$ AcUr: $r=0,53$, $p<0,05)$. Ao analisar as correlações no $G 2$, houve correlação entre IMC, PCR-us $(r=0,66, p<0,01)$ e o ácido úrico $(r=0,37, p<0,01)$; e entre a circunferência da cintura, $P C R$-us $(r=0,40, p<0,05)$ e ácido úrico $(r=0,41, p<0,05)$, segundo a Tabela 3 .
No modelo de regressão logística multivariada, avaliando a glicemia de jejum alterada como variável dependente e as variáveis IMC, CC, PCR-us, AcUr e Hcy como variáveis independentes, observa-se que a CC foi a única variável preditora significante $(p<0,001)$, de acordo com a Tabela 4 .

\section{I S C U S S Ã O}

Além de ser considerado fator de risco independente para as doenças cardiovasculares, o diabetes mellitus tipo 2 triplica o risco de óbito. 0 aumento das concentrações glicêmicas pode estar associado às alterações da composição corporal e de variáveis bioquímicas ${ }^{18}$.

Neste estudo foi verificado que a influência da adiposidade na alteração das concentrações glicêmicas foi mais significante pela adiposidade localizada na região abdominal do que pelo Índice de Massa Corporal total. Além disso, na regressão logística multivariada a variável IMC não teve valor preditivo para glicemia de jejum alterada, fato encontrado somente em relação à circunferência de cintura. Em 2004, Janssen et al..$^{19}$ verificaram que, isoladamente, o IMC e a CC predizem doenças associadas à obesidade. Entretanto, quando avaliados conjuntamente, somente a CC apresentou associação positiva e significante às doenças.

A presença acentuada de gordura na região abdominal (visceral) é um dos principais marcadores biológicos de RI. Wong et al. ${ }^{20}$ observaram que em idosos o IMC e a CC estão inversamente associados à mortalidade em adultos e idosos, mas positivamente ao desenvolvimento do DM2. 
As concentrações de Hcy tenderam a aumentar nos indivíduos com maior IMC na presença de alteração da glicemia de jejum, mas sem significância. Alguns estudos também não observaram associação entre a Hcy e a RI. A razão para estes achados pode ser um aumento na excreção renal de Hcy ou dos seus metabólitos por hiperfiltração glomerular, usualmente presente em indivíduos obesos ${ }^{21-23}$.

Concentrações elevadas de ácido úrico têm sido associadas à maior produção de espécies reativas do oxigênio e do nitrogênio, e ambas à ativação de citocinas pró-inflamatórias por meio da ativação do fator nuclear kappa B, via peróxido de hidrogênio $\left(\mathrm{H}_{2} \mathrm{O}_{2}\right)$ e óxido nítrico $(\mathrm{NO})^{5,7}$. Neste estudo, as concentrações de AcUr foram associadas ao excesso de peso e à CC, sem influência na glicemia de jejum. Taniguchi et al. ${ }^{24}$ obtiveram o mesmo resultado em homens japoneses, concluindo que concentração elevada de AcUr não constitui fator de risco para diabetes tipo 2, mas sim para a síndrome metabólica e o risco cardiovascular25,26.

O estresse inflamatório que tem sido associado tanto ao excesso de peso quanto ao estado de resistência à insulina ${ }^{8}$, neste estudo apresentou correlação positiva apenas nos obesos com glicemia de jejum alterada. Segundo Moran et al. ${ }^{27}$ a relação positiva entre PCR-us e obesidade, fator de risco para o desenvolvimento da $\mathrm{Rl}$, sugere que a associação da PCR-us à RI exista pela presença da adiposidade abdominal. Além disso, a RI pode iniciar ou contribuir com a elevação de PCR-us por redução da supressão induzida pela insulina sob as proteínas reativas hepáticas de fase aguda ${ }^{28}$.

De acordo com o estudo, os estados pró-oxidativo e pró-inflamatório estiveram associados à glicemia de jejum alterada na presença de excesso de peso e gordura abdominal. No modelo de regressão logística, a CC foi a variável explicativa da alteração da glicemia, mas com baixa representatividade (11\%). Isto pressupõe que a alteração da glicemia também mantém relação com os outros fatores analisados, como o inflamatório, fato evidenciado em outros estudos e nas correlações parciais do presente estudo 29,30 .
Estes resultados sugerem que a redução da adiposidade corporal, principalmente a abdominal, seria uma alternativa plausível para reduzir a resistência à insulina, bem como para redução das concentrações de PCR-us e AcUr. Dessa forma, em continuidade ao estudo serão planejadas estratégias de intervenção específicas e adequadas, visando a mudanças na composição corporal, para controle da situação atual e prevenção de futuros problemas.

\section{COLABORADORES}

J.F. MOTA: responsável pela condução da pesquisa, pela avaliação nutricional dos indivíduos participantes, pelas análises estatísticas e pela redação do manuscrito. W.L. MEDINA: responsável pela triagem médica dos indivíduos, participou da análise dos dados e da redação do manuscrito. F. MORETO: responsável pelas análises bioquímicas, participou da redação do manuscrito. R.C. BURINI: orientador e responsável pelo projeto de pesquisa e pela revisão das análises estatísticas, colaborou com a redação final do trabalho.

\section{REFERÊ NCIAS}

1. Sriraman R, Tooke JE. Endothelial dysfunction and insulin resistance. Metab Synd Relat Disord. 2004; 2(2):129-36.

2. Evans JL, Goldfine ID, Maddux BA, Grodsky GM. Are oxidative stress-activated signaling pathways mediators of insulin resistance and $\beta$-cell dysfunction? Diabetes. 2003; 52(1):1-8.

3. Ceriello A. New insights on oxidative stress and diabetic complications may lead to a "causal" antioxidant therapy. Diabetes Care. 2003; 26(5): 1589-96.

4. Coppola A, Astarita C, Oliviero M, Fontana D, Picardi G, Esposito K, et al. Impairment of coronary circulation by acute hyperhomocysteinemia in type 2 diabetic patients. Diabetes Care. 2004; 27(8): 2055-6.

5. Hayden MR, Tyagi SC. Uric acid: a new look at an old risk marker for cardiovascular disease, metabolic syndrome, and type 2 diabetes mellitus: the urate redox shuttle. Nutr Metab. 2004; 1(10): $1-10$.

6. Kanellis J, Watanabe S, Li JH, Kang DH, Li P, Nakagawia $\mathrm{T}$, et al. Uric acid stimulates monocyte 
chemoattractant protein-1 production in vascular smooth muscle cells via mitogen-activated protein kinase and cyclooxygenase-2. Hypertension. 2003; 41(6):1287-93.

7. Olexa P, Olexova M, Gonsorcik J, Tkac I, Kisel'ova J, Olejnikova M. Uric acid: a marker for systemic inflammatory response in patients with congestive heart failure? Wien Klin Wochenschr. 2002; 114 (5-6):211-5.

8. Pradhan AD, Manson JE, Rifai N, Buring JE, Ridker PM. C-reactive protein, interleukin 6, and risk of developing type 2 diabetes mellitus. Jama. 2001; 286(3):327-34.

9. Ridker PM. High-sensitivity C-reactive protein and cardiovascular risk: rationale for screening and primary prevention. Am J Cardiol. 2003; 929(4B): $17 k-22 k$.

10. Sutherland JP, McKinley B, Eckel RH. The metabolic syndrome and inflammation. Metab Syndr Relat Disord. 2004; 2(2):82-104.

11. Kolb K, Mandrup-Poulsen T. An immune origin of type 2 diabetes? Diabetologia. 2005; 48(6): 1038-50.

12. Festa A, D'Agostino Jr R, Hanley AJG, Karter AJ, Saad MF, Haffner SM. Differences in insulin resistance in nondiabetic subjects with isolated impaired glucose tolerance or isolated impaired fasting glucose. Diabetes. 2004; 53(6):1549-55.

13. Fonseca-Alaniz MH, Takada J, Alonso-Vale MIC, Lima BF. O tecido adiposo como centro regulador do metabolismo. Arq Bras Endocrinol Metab. 2006; 50(2):216-28.

14. American Diabetes Association. Diagnosis and classification of diabetes mellitus. Diabetes Care. 2005; 28(1):S37-S42.

15. World Health Organization. Diet, nutrition and the prevention of chronic diseases. Geneva: WHO; 2002.

16. Friedewald TW, Levy RI, Friederickson DS. Estimation of the concentration of low density lipoprotein cholesterol in plasma, without the use of the preparative ultracentrifuge. Clin Chem. 1972; 18(6):499-502.

17. Stanger O, Herrmann W, Pietrzik K, Fowler B, Geisel J, Dierkes J, et al. DACH- LIGA homocystein (German, Austrian and Swiss homocysteine society): Consensus paper on the rational clinical use of homocysteine, folic acid and B-vitamins in cardiovascular and thrombotic diseases: guidelines and recommendations. Clin Chem Lab Med. 2003; 41(11):1392-403.

18. Salles JF, Bloch KV, Cardoso CRL. Mortality and predictors of mortality in a cohort of Brazilian type 2 diabetic patients. Diabetes Care. 2004; 27(6): 1299-305.
19. Janssen I, Katzmarzyk PT, Ross R. Waist circumference and not body mass index explains obesity-related health risk. Am J Clin Nutr. 2004; 79(3):379-84.

20. Wong SL, Janssen I, Ross R. Abdominal adipose tissue distribution and metabolic risk. Sports Med. 2003; 33(10):709-28.

21. Tanrikulu-Kiliç F, Bekpinar S, Ünlüçerçi Y, Orhan Y. Insulin resistance is not related to plasma homocysteine concentration in healthy premenopausal women. Physiol Res. 2006; 55(3):285-90.

22. Schäfer SA, Müssig K, Stefan N, Häring HU, Fritsche A, Balletshofer BM. Plasma homocysteine concentrations in young individuals at increased risk of type 2 diabetes are associated with subtle differences in glomerular filtration rate but not with insulin resistance. Exp Clin Endocrinol Diabetes. 2006; 114(6):306-9.

23. Hall JE, Brands MW, Henegar JR. Mechanism of hypertension and kidney disease in obesity. Ann N Y Acad Sci. 1999; 892(11):91-107.

24. Taniguchi Y, Hayashi T, Tsumura K, Endo G, Fujii S, Okada K. Serum uric acid and the risk for hypertension and Type 2 diabetes in Japanese men: the Osaka Health Survey. J Hypertens. 2001; 19(7):1209-15

25. Niskanen LK, Laaksonen DE, Nyysönen K, Alfthan G, Lakka HM, Lakka TA, et al. Uric acid level as a risk factor for cardiovascular and all-cause mortality in middle-aged men: a prospective cohort. Arch Intern Med. 2004; 164(14):1546-51.

26. Baker JF, Krishnan E, Chen L, Schumacher HR. Serum uric acid and cardiovascular disease: recent developments, and where do they leave us? Am J Med. 2005; 118(8):816-26.

27. Moran A, Steffen LM, Jacobs DR Jr, Steinberger J, Pankow JS, Hong CP, et al. Relation of C-reactive protein to insulin resistance and cardiovascular risk factors in youth. Diabetes Care. 2005; 28(7): 1763-8.

28. Campos WP, Baumann $\mathrm{H}$. Insulin is a prominent modulator of the cytokine stimulated expression of acute-phase plasma protein genes. Mol Cell Biol. 1992; 12(4):1789-97.

29. Mota JF, Medina WL, Moretto F, Burini RC. Nutritional and metabolic determinants of insulin resistance in adults. FASEB J. 2007; 21(837.4): A1068.

30. Rabe K, Lehrke M, Parhofer KG, Broedl UC. Adipokines and insulin resistance. Mol Med. 2008; 14(11-12):741-51.

Recebido em: 19/1/2007

Versão final reapresentada em: 3/12/2008 Aprovado em: 9/3/2009 